\title{
Novel insight into the potential pathogenicity of mitochondrial dysfunction resulting from PLP1 duplication mutations in patients with Pelizaeus- Merzbacher disease
}

\section{Ruoyu Duan}

Peking University First Hospital

Liuju Li

Peking University

Huifang Yan

Peking University First Hospital

Miao He

South China Normal University

Kai Gao

Peking University First Hospital

Shijia Xing

Peking University

Haoran Ji

Peking University First Hospital

Jianyong Wang

Peking University

\section{Binbin Cao}

Peking University

Dongxiao Li

Peking University

Han Xie

Peking University First Hospital

Shiqun Zhao

Peking University

\section{Ye Wu}

Peking University First Hospital

\section{Yuwu Jiang}

Peking University First Hospital

Jiangxi Xiao 


\section{Qiang Gu}

Peking University First Hospital

\section{Ming Li}

Peking University First Hospital

\section{Xiaolu Zheng}

Peking University

\section{Liangyi Chen}

Peking University

\section{Jingmin Wang ( $\square$ wang66jm@163.com )}

Peking University First Hospital https://orcid.org/0000-0001-5263-1013

\section{Research Article}

Keywords: Pelizaeus-Merzbacher disease (PMD), PLP1

Posted Date: February 9th, 2021

DOI: https://doi.org/10.21203/rs.3.rs-216270/v1

License: (c) (i) This work is licensed under a Creative Commons Attribution 4.0 International License. Read Full License 


\section{Abstract}

Among the hypomyelinating leukodystrophies, Pelizaeus-Merzbacher disease (PMD) is a representative disorder. The disease is caused by different types of PLP1 mutations, among which PLP1 duplication accounts for $\sim 70 \%$ of the mutations. Previous studies have shown that PLP1 duplications lead to PLP1 retention in the endoplasmic reticulum (ER); in parallel, recent studies have demonstrated that PLP1 duplication can also lead to mitochondrial dysfunction. As such, the respective roles and interactions of the ER and mitochondria in the pathogenesis of $P L P 1$ duplication are not clear. In both PLP1 patients' and healthy fibroblasts, we measured mitochondrial respiration with a Seahorse XF Extracellular Analyzer and examined the interactions between the ER and mitochondria with super-resolution microscopy (spinning-disc pinhole-based structured illumination microscopy, SD-SIM). For the first time, we demonstrated that PLP1 duplication mutants had closer ER-mitochondrion interfaces mediated through structural and morphological changes in both the ER and mitochondria-associated membranes (MAMs). These changes in both the ER and mitochondria then led to mitochondrial dysfunction, as reported previously. This work highlights the roles of MAMs in bridging PLP1 expression in the ER and pathogenic dysfunction in mitochondria, providing novel insight into the pathogenicity of mitochondrial dysfunction resulting from PLP1 duplication. These findings suggest that interactions between the ER and mitochondria may underlie pathogenic mechanisms of hypomyelinating leukodystrophies diseases at the organelle level.

\section{Introduction}

Hypomyelinating leukodystrophies (HLD) is a large category of neurological disorders with a distinct characteristic of hypomyelination, among which Pelizaeus-Merzbacher disease (PMD; MIM 312080) is representative. $\mathrm{PMD}$ is a rare $\mathrm{X}$-linked hypomyelination disorder caused by mutations in the proteolipid protein 1 gene ( $P L P 1$, NM_001128834.20), which is located on Xq21-q22 and encodes two major central nervous system (CNS) myelin proteins, including PLP1 and its spliced isoform DM20. Its clinical manifestations are nystagmus, hypotonia, ataxia, athetotic movements, and cognitive impairment, among others [1]. Based on the clinical severity, PMD can be classified into the connatal, transitional, and classical types. At the time of this work, our group had accumulated 94 PMD cases [2], giving us a unique opportunity to investigate the pathogenic mechanisms of this rare genetic disorder.

Previous studies have shown different pathogenic mechanisms resulting from point and duplication mutations of PLP1 [3-5], respectively. Point mutations lead to misfolded protein accumulation in the endoplasmic reticulum (ER), triggering an unfolded protein response (UPR) pathway or defective trafficking and fusion of mutated PLP1 [5]. A larger proportion of the cases, however, have been associated with duplication mutations: almost $70 \%$ of PMD cases can be attributed to PLP1 duplication and approximately $25 \%$ to point mutations [6, 7]. Our patient pool also supports this distribution with 65 patients carrying PLP1 duplications and 29 carrying PLP1 point mutations [2]. 
Duplication mutations of $P L P 1$ lead to toxic overexpression of its protein, resulting in clinical manifestations of the disease. Overexpression of PLP1 has been reported in the skin fibroblasts of PMD patients with $P L P 1$ duplication [8], yet the effects of $P L P 1$ duplication on the cellular processes are still not clear. Few studies have addressed this question. In a PLP1 duplication mutation mouse model, Ruiz et al. recently found abnormal intracellular ATP levels and mitochondrial membrane potentials [9]. The study by Miyamoto et al. has confirmed the shortening of mitochondria in another type of hypomyelinating leukodystrophies, leading to mitochondrial dysfunction [10]. These studies indicate the roles of mitochondrial dysfunction in PLP1-related diseases.

The mitochondrion is an important bilayer membrane organelle that plays important role in numerous essential biosynthetic pathways [11-13]. However, limited studies have linked PLP1 overexpression in the endoplasmic reticulum (ER) to mitochondrial morphology and functions. Previous studies have suggested that PLP1 is expressed in the ER $[14,15]$. It remains unclear how interactions of the ER and mitochondria can result in disease. In Alzheimer patients' fibroblasts, upregulation of mitochondriaassociated membranes (MAMs) function and significant increases in ER-mitochondrial connectivity were found to alter lipid metabolism and calcium homeostasis [16]. Therefore, it is possible that MAMs mediate the mitochondrial dysfunction. MAMs play important roles in mediating cellular processes including mitochondrial division, calcium homeostasis, and apoptotic signaling [17-20]. They serve as a bridge, which is decorated by ER to form a contact covering 10\%- 50\% of the mitochondria surface [21, 22]. Therefore, MAMs may be the key factor underlying the interactions of ER and mitochondria in the pathogenicity of PLP1 duplication.

Our understanding of mitochondria has been based on optical and electron microscopes. It has been difficult to clearly image interactions of organelles in live cells because of sample fixation problems and limited spatial resolution. However, the emergence of superresolution (SR) fluorescence microscopy in the 21 st century has made superresolution live cell imaging possible. This method provides new insights into intricate intracellular structures and processes $[23,24]$. Moreover, it reduces photon doses, making it more living sample-friendly due to reduced photobleaching and phototoxicity. SR microscopy can also be used to capture dynamic activity in live cells during disease pathogenesis [23]. In this work, we propose to use state-of-the-art superresolution microscopy to uncover the potential pathogenetic ER-mitochondrial interactions.

The aim of this study is to explore the potential pathogenicity of mitochondrial dysfunction resulting from PLP1 duplication mutations. Our hypothesis is that overexpression of PLP1 in the ER affects the MAM structure, resulting in mitochondrial dysfunction and downstream cytotoxicity of PLP1. To test this hypothesis, we studied patients' fibroblast using immunocytochemistry. We imaged ER-mitochondrial interactions using a recently developed SR microscope, and we measured mitochondrial respiration function with Seahorse XF Extracellular Flux. To the best of our knowledge, this is the first report to focus on the interaction between organelles to demonstrate the pathogenesis of mitochondrial dysfunction in PMD patients carrying a PLP1 duplication. The results from this study could provide a better 
understanding of the potential pathogenicity of mitochondria dysfunction resulting from PLP1 duplication mutations in Patients with Pelizaeus-Merzbacher disease.

\section{Materials And Methods}

\section{Patients}

From our cohorts of patients (Pt1, Pt2, Pt3), we selected three male patients aged 13 year 1 mo, 9 year 3 mo and 8 year 9 mo with representative classical or connatal PMD types (Table 1). This study was approved by the local ethics committees of Peking University First Hospital. Written informed consent was acquired from the patients' parents or legal guardians. A modified phenotypic classification score (PCS) index from previous studies $[25,26]$ was used to investigate the clinical severity of PMD for each of the patients. The PCS values range from $0-5$, with values from 0 to 2 representing severe phenotypes and values from 3 to 5 representing mild phenotypes. Combined with patients' brain MRI scans, their clinical subtype phenotypes can be concluded. For the patients' genetic diagnosis, we performed PLP1 multiplex ligation-dependent probe amplification (MLPA) using an assay kit (P071, MRC Holland, Netherlands).

\section{Cell culture and transfection}

Fibroblasts were isolated from the left forearms of the three PMD patients and cultured in high-quality medium composed of F12 (8117165, Gibco, CA, USA), 20\% fetal bovine serum (FBS, 10099, Gibco, CA, USA), and 1\% PS (15140-122, Gibco, CA, USA). The cell cultures were obtained by adhesion and expanded to maximum confluency after 15 passages in $25-\mathrm{cm}^{2}$ flasks. The flasks were kept in an environment of $5 \% \mathrm{CO}^{2}$ and $37^{\circ} \mathrm{C}$. To avoid in vitro biases, the fibroblasts in this experiment were only used after the cell cultures reached at least $80 \%$ confluency. Transfection was performed by electroporation (Neon® Transfection System, Invitrogen, CA, USA); $0.5 \mu \mathrm{g}$ Mito-YFP and $1 \mu \mathrm{g}$ mCherry-Sec $61 \beta$ Plasmid-DNA [27] were added to $1.5-2 \times 10^{6}$ cells in $10 \mu$ l plating medium (MPK1025, Invitrogen, CA, USA). Electroporation parameters were optimized to voltage $=165 \mathrm{~V}$, width $=10 \mathrm{MA}$, and pulse $=3$. Cells were stained with Dulbecco's Modified Eagle Medium (DMEM), $20 \% \mathrm{FBS}, 5 \%$ and $\mathrm{CO}_{2}$ at $37^{\circ} \mathrm{C}$ for $24-36$ hours before the subsequent experiments.

\section{Mitochondrial respiration}

The oxygen consumption rate $(\mathrm{OCR})$ was used to measure real-time mitochondrial respiration in the fibroblasts with a Seahorse XF Extracellular Flux Analyzer. After 24-36 hours, to assess mitochondrial respiration, three modulators (102353-100, Agilent, CA, USA) were added to the cell culture at different stages of the electron transport chain (ETC) according to Divakaruni et al.'s study [28] (Fig. 1a). First, 1 $\mu \mathrm{M}$ oligomycin was added to inhibit ATP synthase (complex V), resulting in a reduction in mitochondrial respiration. Then, $1 \mu \mathrm{M}$ carbonyl cyanide-4 (trifluoromethoxy) phenylhydrazone (FCCP) was added to collapse the proton gradient, leading to uninhibited electron flow and maximal OCR. Finally, a mixture of 
$0.5 \mu \mathrm{M}$ rotenone (complex I inhibitor) and $0.5 \mu \mathrm{M}$ antimycin A (complex III inhibitor) was injected to shut down mitochondrial respiration.

Cells were divided into patients and controls obtained from PLP1 duplication patients and normal subjects, respectively. After 16-24 hours, cells were seeded in XF cell culture microplates (100867-100,

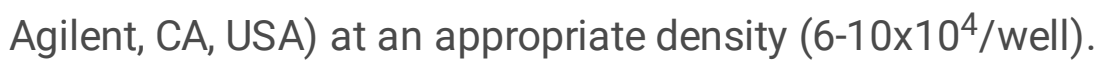

Basal respiration and maximal respiration rates were calculated from the analysis of the OCRs in this study $[29,30]$. To exclude differences in the numbers of cells between groups, the basal respiration rate was defined as the average OCR value at baseline prior to the addition of any modulation, which was measured at the start of this experiment; the maximal respiration rate was attained by adding FCCP, which can mimic a physiological "energy demand" by stimulating the respiratory chain to operate at the maximum capacity, causing rapid oxidation of substrates (sugars, fats, amino acids) to meet its metabolic challenge. The maximum respiration rate was the maximal OCR that the cell could achieve. The OCR (pMoles/min) was measured five times at twelve different time points; the average value from each time point was used for statistical analysis. Three parallel experiments were conducted for each cell line. The basal and maximal respiration rates were used as key indicators of mitochondrial function for the subsequent analyses.

\section{Immunofluorescence}

To investigate the localization of PLP1 inside the cell, cells were first fixed with 4\% paraformaldehyde (Leagene, DF0135, Beijing, China) for 10 minutes at room temperature (RT, 20 ${ }^{\circ} \mathrm{C}$ ). After 24-36 hours of culture, cells were rinsed with PBS (RNBH2442, Sigma, St. Louis, MO, USA) three times for 5 min each, permeabilized with $0.3 \%$ Triton for 10 min, and again rinsed with PBS three times for 5 min each. After being blocked with 5\% BSA (Solarbio, A8010, Beijing, China) for $30 \mathrm{~min}$, the cells were incubated with an anti-PLP1 rabbit polyclonal antibody (Cat: 102284-T08, Sino Biological, Beijing, China) at a 1:500 dilution in PBS overnight at $4^{\circ} \mathrm{C}$. After 12-16 hours, the cells were rinsed with PBS three times and incubated with goat anti-rabbit $(\mathrm{H}+\mathrm{L})$ Alexa Fluor 405 (A-31556, Invitrogen, CA, USA) secondary antibody diluted 1:1000 in PBS for $1 \mathrm{~h}$ at RT. Finally, the cells were rinsed with PBS three times.

\section{Superresolution live cell imaging}

Fibroblasts were cultured in an adhesive chamber with glass coverslips at the bottom. In all live-cell imaging experiments, cells were incubated in complete medium inside a closed chamber at $37^{\circ} \mathrm{C}$.

After preparing the cells for live imaging, they were then imaged by SD-SIM. The SD-SIM setup was a commercial system based on an inverted fluorescence microscope (Olympus, IX81) equipped with a 100x, 1.3 numerical aperture (NA) oil objective and a scanning confocal system (Yokogawa, CSU-X1). Three laser beams with wavelengths of $488 \mathrm{~nm}, 561 \mathrm{~nm}$, and $405 \mathrm{~nm}$ were used in this study. The LiveSR module (GATACA systems, France) was equipped with SD-SIM [31, 32]. The images were acquired githar hy an EMAnn (Andar iVnn2 Q07) ar a anMnnc (Unmamatsu, C14440-20UP) camera. The Loading [MathJax]/jax/output/CommonHTML/fonts/TeX/fontdata.js 
extracellular Hank's balanced salt solution (HBSS, 14025076, Gibco, CA, USA) was maintained at temperatures between 30 and $37^{\circ} \mathrm{C}$.

\section{Western blotting}

Fibroblasts were harvested with RIPA buffer (50 mM Tris-HCl, $1 \%$ Nonidet P- $40,150 \mathrm{mM} \mathrm{NaCl}, 0.5 \%$ sodium deoxycholate, $0.1 \%$ SDS and complete protease inhibitor, $1 \mathrm{mM}$ EDTA). Fifty to one hundred micrograms of protein was loaded into each well in a $10 \%$ gel. After the proteins were transferred onto a PVDF membrane and blocked with $5 \%(\mathrm{w} / \mathrm{v})$ nonfat milk for $1 \mathrm{~h}$, they were incubated with primary antiPLP1 antibody (Cat: 102284-T08, Sino Biological, Beijing, CHN) and anti- $\beta$-actin (C1313, APPLYGEN, $\mathrm{CHN}$ ) diluted 1:1000 overnight at $4^{\circ} \mathrm{C}$. The blots were washed 3 times and then probed with secondary antibody (goat anti-rabbit HRP, C1309, APPLYGEN, CHN/goat anti-mouse HRP, C1308, APPLYGEN, CHN) conjugate diluted 1:3000 for $1 \mathrm{~h}$. After washing, the protein expression was detected (WBKLS0100, Millipore, chemiluminescent HRP Substrate, USA).

\section{IStatistical analysis}

All experiments were conducted at least three times. Statistical analyses were performed with Fiji and Prism6 (GraphPad software). Images of immunofluorescence and SD-SIM were imported into Fiji, an open-source Image $J$ version for processing images, which also contains preinstalled plugins such as coloc2 for measuring the colocalization coefficients to calculate the interface between the ER and mitochondria. Differences between groups were assessed by analysis of variance (ANOVA) or the unpaired Student's $t$ test; the significance level was set at $p<0.05$.

\section{Results}

\section{Clinical and genetic results}

The patients were diagnosed with PMD based on their clinical assessment and MRI scans. The disease onset for the three patients was $2 \mathrm{mo}, 5 \mathrm{mo}$ and $1 \mathrm{mo}$ (Table 1). None of the patients presented a family history. All patients showed nystagmus and developmental delays, including signs of cognitive and psychomotor delays (Table 1). All of them had ataxia, which contributed to their motor impairment. Other neurological disorders, such as seizures or spastic paraplegia, were ruled out from the clinical assessment. MRI scans further confirmed patients' PMD diagnosis. All three patients' MRI scans were similar to that of Pt1, whose white matter hypomyelination was observed, as shown in Fig. S1. Diffuse hyperintensity in white matter presented with a high signal on the axial T2WI scans at 1 year, 2 year and 4 year (Fig. S1, bottom row). Based on the clinical severity and MRI, all three patients were diagnosed with PMD. Pt2 was classified as connatal type (PCS $=1)$; correspondingly, Pt1 (PCS $=4)$ and Pt3 (PCS = 3) were the classical type. In addition to the clinical assessment and MRI scan, genetic diagnosis was used to confirm the PMD diagnosis. The results of $P L P 1$ multiplex ligation-dependent probe amplification (MLPA) showed that they carried different PLP1 duplication fragments. 
Table1: Clinical and genetic information of the three patients

\begin{tabular}{|c|c|c|c|c|c|c|c|c|c|c|c|c|}
\hline \multirow{2}{*}{ Subject } & \multirow{2}{*}{ Sex } & \multirow{2}{*}{ Age } & \multirow{2}{*}{ PCS } & \multirow{2}{*}{$\begin{array}{c}\text { Disease } \\
\text { Onset }\end{array}$} & \multicolumn{4}{|c|}{ Psychomotor Development } & \multirow{2}{*}{ Fam } & \multirow{2}{*}{ Type } & \multirow{2}{*}{$\begin{array}{l}\text { Duplication } \\
\text { fragments }\end{array}$} & \multirow{2}{*}{ Origin } \\
\hline & & & & & Head & Sit & Stand & Speak & & & & \\
\hline Pt1 & $\mathbf{M}$ & 13yr1mo & 4 & $2 \mathrm{mo}$ & $3 \mathrm{mo}$ & $6 \mathrm{mo}$ & - & $30 \mathrm{mo}$ & - & classical & $1.8 \mathrm{Mb}$ & Matemal \\
\hline$P 12$ & $\mathbf{M}$ & $9 y r 3 m o$ & 1 & $5 \mathrm{moo}$ & $21 \mathrm{mo}$ & - & - & - & - & connatal & $1.2 \mathrm{Mb}$ & Matemal \\
\hline Pt3 & $\mathbf{M}$ & $8 y r 9 m o$ & 3 & Imo & $8 \mathrm{mo}$ & $12 \mathrm{mo}$ & - & - & - & classical & $801 \mathrm{~Kb}$ & Matemal \\
\hline
\end{tabular}

M:male. Head:onset of rising one's head steadily. Sit/Stand: onset of sitting/standing on their own. Speak: onset of speaking a few Chinese words. Fam $\rrbracket$ family history of PMD. yr: year. mo: month. +: positive; -: not acquiring the ability or not having a family history.

\section{Mitochondrial respiration levels are reduced in PLP1-duplicated fibroblasts}

We used a Seahorse XF Extracellular Flux Analyzer to monitor the mitochondrial oxygen consumption rate (OCR). The mechanism of the mitochondrial respiration experiment is illustrated in Fig. 1a. The basal respiration-OCR was the average OCR at 0 min prior to the addition of the three electron transport chain interrogation drugs, as shown in Fig. 1c. The control subject's cells had a basal OCR rate with an average level of $316.99 \pm 89.45 \mathrm{pMoles} / \mathrm{min}$. There were no significant differences $(p>0.05)$ between the control subject's basal OCR and those of the three patients, which were $257.45 \pm 55.81 \mathrm{pMoles} / \mathrm{min}, 235.27 \pm$ $142.63 \mathrm{pMoles} / \mathrm{min}$, and $305.12 \pm 46.68 \mathrm{pMoles} / \mathrm{min}$, respectively. However, the differences were not statistically significant (Fig. 1c, $p>0.05$ ). For maximal OCR, the three patients' average maximal OCRs were $307.79 \pm 85.10 \mathrm{pMoles} / \mathrm{min}, 265.69 \pm 159.90 \mathrm{pMoles} / \mathrm{min}$, and $375.27 \pm 101.60 \mathrm{pMoles} / \mathrm{min}$, respectively. The control subject's average maximal OCR was significantly higher than the patients' average maximal OCR (Fig. 1d, $p<0.05$ ) at $459.76 \pm 113.22 \mathrm{pMoles} / \mathrm{min}$. Among the three patients, the maximal OCR of Pt3 was highest and Pt2 was lowest (Fig. 1b), but there were no differences among the three patients. These results may be consistent with the patients' clinical severity because Pt3 was diagnosed with the classical sub PMD type, which was less severe than Pt2's diagnosis of the connatal subtype.

\section{Accompanied by PLP1 colocalization with the ER, the peripheral ER displays more "expanded sheet"-like structures}

As shown in Fig. 2a, PLP1 mainly colocalized with the ER rather than mitochondria. Moreover, in PLP1duplicated fibroblasts, some "puncta" structures could be seen in the regions where PLP1 colocalized with the ER, accounting for nearly $70 \%$ of the colocalization regions; in contrast, puncta structures could hardly be detected in the control fibroblasts. Because the distribution of PLP1 was more uniform than that of SEC61, an ER membrane protein for labeling the ER structure (Fig. 2a), we suspected that these structures were more similar to the ER structures. When the ER was magnified, both control and PLPLduplicated fibroblasts showed peripheral ER domains consisting of perinuclear ER sheets and tubules (Fig. $2 \mathrm{~b}$ and 2c). The patients' fibroblasts, however, had more ER sheets in the peripheral ER, accounting for over $60 \%$ of patients' fibroblasts as opposed to only $10 \%$ of the control fibroblasts (Fig. $2 \mathrm{c}$ ). The greater fraction of ER sheets in patient fibroblasts was accompanied by decreases in the counts of 
$\mu \mathrm{m}^{2}, 207.91 \pm 113.01 \mu \mathrm{m}^{2}$, and $160.12 \pm 80.70 \mu \mathrm{m}^{2}$, exceeding those of control subject fibroblasts measuring $30.38 \pm 36.23 \mu \mathrm{m}^{2}$. Therefore, $P L P 1$ duplication fibroblasts had significantly more expanded sheets (Fig. $2 b$ and $2 d, p<0.01$ ) among the peripheral ER.

\section{PLP1 duplication fibroblasts show a higher level of ER-mitochondria interfaces near the expanded ER sheet}

We then investigated whether the abnormal ER distribution and structure altered the ER and mitochondrial interface by examining areas close to the expanded ER sheets, as shown in the enlarged views of Fig. 3a. The colocalization coefficients of PLP1 duplication fibroblasts were greater than those of the control (Fig. 3b, $p<0.01$ ), among which Pt2 had the highest colocation coefficient of $\sim 40 \%$. In comparison, the control subject's colocation coefficients were only $10 \%$. suggesting that a larger section in the channel of the ER colocalizes with mitochondria in patient fibroblasts. Therefore, PLP1 duplication mutant fibroblasts had significantly more interfaces between expanded ER sheets and mitochondria.

\section{PLP1 duplication patients' mitochondria show an abnormal morphology and shorter lengths}

Based on the study by Jian et al. [34], the mitochondrial morphology we observed in our experiment could be divided into four types: tubular, short tubular, fragmented and large spherical (LASMs) (Fig. 4a). Compared with the control fibroblasts, the $P L P 1$ duplication fibroblasts' mitochondrial morphology was abnormal, with a smaller percentage of the tubular shape $(p<0.05)$ and conversely greater percentages of the short tubular, fragment, and LASM shapes $(p<0.05)$ (Fig. 4b). Among the patients, Pt3 had less of the tubular shape than Pt1 and Pt2, and Pt2 had the maximum percentage of the LASM shape (Fig. 4b, P $>0.05$ ). Consistent with the morphology shown in Fig. $4 \mathrm{~b}$, the patients' mitochondria were significantly shorter than those of the controls (Fig. 4c, p < 0.01). To illustrate, the lengths of patients' fibroblast mitochondria were measured at $4.81 \pm 2.91 \mu \mathrm{m}, 3.88 \pm 1.52 \mu \mathrm{m}$, and $4.04 \pm 2.01 \mu \mathrm{m}$, respectively, compared with the control fibroblasts measured at $8.24 \pm 3.93 \mu \mathrm{m}$.

\section{PLP1 expression is higher in patient than control patient fibroblasts}

Based on the western blotting results (Fig. S2), we showed that PLP1 expression in control and patients' fibroblasts was $4.15 \pm 3.95(\%), 14.07 \pm 12.57$ (\%), $12.54 \pm 7.13(\%)$, and $20.65 \pm 6.00$ (\%). Based on these results, PLP1 expression was higher in patient than control fibroblasts. No differences were found in PLP1 expression between Pt1 and Pt2 and the control group ( $n=3 p>0.05)$, although a significant difference was detected between Pt3 $(n=3 p<0.05)$ and the control group (Fig. S2b).

\section{Discussion}

PMD presents a wide range of symptoms and complications caused by a deficit of myelin deposits in the white matter. Hypotonia, nystagmus, spastic quadriparesis, ataxia, extrapyramidal signs, and hypomyelination are commonly manifested on MRI [15]. The clinical diagnosis of PMD is based on 
characteristics including delayed motor milestones, hypotonia, nystagmus, and hypomyelination on MRI. According to the phenotypic classification (PCS) score (Table 1), Pt2 was classified as the connatal type $(P C S=1)$; correspondingly, Pt1 (PCS = 4) and Pt3 (PCS = 3) were the classical type. Genetic testing showed that Pt1-Pt3 carried a PLP1 duplication. Research has shown that the PLP1 duplication can increase the transcript level of PLP1, resulting in its cellular overexpression [8]. Our study confirmed that PLP1 expression was higher in patient than control fibroblasts (Fig. S2). However, the cellular and molecular mechanisms underlying the phenotype in PMD patients with a PLP1 duplication was unclear.

A study by Ruiz et al. associated PLP1 overexpression with the dysfunction of mitochondrial respiratory functions [9]. Mitochondria play important roles in bioenergy and cell biology pathways, and their predominant physiological function is the generation of ATP through oxidative phosphorylation. The mitochondrial proton circuit across the inner membrane is crucial to mitochondrial respiration. To evaluate mitochondrial respiratory function, mitochondrial proton currents were assessed by the oxygen consumption rate (OCR), which was measured using oxygen-dependent fluorescence quenching [35]. A lower maximum OCR may result from compromised mitochondrial integrity. A higher maximum OCR correlates with acute impairment [36-38]. Brand et al. suggested that a decrease in maximum OCR may be a strong indicator of potential mitochondrial dysfunction [35]. Our results showed that the maximal OCR was lower in fibroblast mitochondria of the PLP1 duplication mutants than the control group (Fig. 1d), which indicated that PLP1 duplication mutants might have a compromised mitochondrial integrity and impaired mitochondrial respiratory function. A previous study obtained similar results in COS-7 cells [39]; they found that compared with nontransfected COS-7 cells, the maximal and basal OCRs were lower in COS-7 cells expressing PLP, but the exact mechanism of mitochondrial dysfunction resulting from the $P L P 1$ duplication remains unclear.

An organelle's structure determines its function to some degree. To understand mitochondrial dysfunction, we linked dysfunction to changes in morphology. A normal mitochondrial morphology can be classified into tubular, short tubular, fragmented, and large spherical mitochondria (LASMs) [34], which were observed in our experiments (Fig. 4a). The tubular conformation has been reported to be maintained by the mitochondrial contact site and cristae organizing system (MICOS) complex, a core component in cristae and crista junctions. Subunits of the MICOS complex, Sam50, Mic19 and Mic60, constitute a subcellular structure known as the Sam50-Mic19-Mic60 axis, which sustains the structure of cristae. Disruption of this axis results in loss of crista junctions and abnormal mitochondrial morphology, consequently leading to mitochondrial dysfunction $[40,41]$.

The depletion of Mic60 or Sam50 knockout causes the formation of LASMs. In our study, there were more LASMs in the mitochondria of the PLP1 duplication mutant than the control (Fig. 4b), demonstrating that the mitochondrial structure could be disrupted. In addition to the abnormal formation of LASMs, we also found that the average length of mitochondria was shorter compared with the control (Fig. 4c). The length of mitochondria may be related to mitochondrial fragmentation, which is the first step in selective autophagosome (mitophagosome) formation or the process of mitochondrial fission [42]. A study has 
DNM1L/DRP1 to target the final scission of mitochondria. As a possible result of mitophagosomes, mitochondrial fragmentation could explain our observation of the shorter average length of mitochondria compared with the control (Fig. 4c).

Taken together, we demonstrated that respiratory functions and mitochondrial structural integrity were impaired in PLP1-duplicated fibroblasts, as evidenced by decreases in OCRs, more LASMs, and shorter average mitochondrial lengths. These results corroborated previous findings regarding the role of mitochondrial respiratory functions in PLP1-related diseases. However, previous studies have indicated that PLP1 is expressed in the endoplasmic reticulum (ER). Therefore, we propose a novel hypothesis that overexpression of PLP1 in the ER affects the mitochondria-associated membrane (MAM) structure, resulting in mitochondrial dysfunction and subsequent cytotoxicity of PLP1. To test our hypothesis, we needed to link these results to the morphology and functions of the ER.

First, we compared the ER morphology in control and PLP1-duplicated fibroblasts. The ER consists of the perinuclear ER and the peripheral ER [43-45]. ER sheets are mainly distributed in perinuclear sites, and only a low percentage of ER sheets can be observed in the periphery. Moreover, most ER tubules are located in the periphery. The ratio of sheets to tubules varies in different processes. In yeasts and COS7 cells, ER sheet rates are higher in translocation protein machinery processes, they may be the preferred site of protein translocation $[46,33,47]$.

In comparison to the control, we found abnormal distributions of ER tubules and sheets in PLP1 duplication fibroblasts. As shown in Fig. 2b, the perinuclear ER in control subject fibroblasts had closed membrane sheets (or cisterna), and the peripheral ER consisted of a network of sheets and tubules. In contrast, in the PLP1 duplication mutant fibroblasts, ER sheets were observed in both the perinuclear and peripheral regions, and more ER sheets were observed in the perinuclear region (Fig. 2c). In addition, the ER sheets exhibited an 'expanded' morphology (Fig. 2d). Furthermore, PLP1 mainly colocalized with the peripheral ER (Fig. 2a), where more ER sheets were located. A study also demonstrated that the peripheral ER generates expanded ER sheets to withstand ER stress [48]. One possible explanation for our observations is that the increasing ER sheet size may provide proteins with more time to fold by avoiding aggregate formation. The increased number of ER sheets in our results might indicate their additional need to address the overexpression of PLP1. Moreover, their expanded morphology suggested that they might be in an abnormal circumstance, for example, protein folding itself. Therefore, the ER sheet distribution and morphology were changed in PLP1 duplication fibroblasts.

To link abnormal ER distributions to mitochondrial dysfunction, we investigated the dynamic structure of MAMs, which serve as a bridge between the ER and mitochondria. ER morphology and location might affect MAM function. A critical function of MAMs is to coordinate $\mathrm{Ca}^{2+}$ leakage from the ER: $\mathrm{Ca}^{2+}$ is first released through $\mathrm{IP}_{3} \mathrm{R}$ from the ER section that is in close contact with mitochondria and then transported through the mitochondrial $\mathrm{Ca}^{2+}$ uniporter (MCU) into mitochondria (Fig. 5) [35]. Therefore, MAM function could be affected by MAM distance, which reflects the expansion of the ER-mitochondrion interfaces. The 
interface explains the amount of overlap between the ER and mitochondria: the higher the level of the interface, the closer is the distance of the MAMs.

For the abnormal 'expanded ER sheets' found in the peripheral ER, we investigated the interface between expanded ER sheets and mitochondria. We used the colocalization coefficient to calculate the extent of the interface between the ER and mitochondria. Greater colocalization coefficients (Fig. 3b) were found near the expanded ER sheets and mitochondria in the PLP1 duplication fibroblasts, which indicated a closer distance between expanded ER sheets and mitochondria. The close distances between the ER and mitochondrial membranes might lead to continuous mitochondrial $\mathrm{Ca}^{2+}$ uptake during background $\mathrm{Ca}^{2+}$ release, which in turn could cause mitochondrial $\mathrm{Ca}^{2+}$ overloading and membrane permeabilization. Moreover, this pathway might trigger IP3-induced $\mathrm{Ca}^{2+}$ mobilization from the ER to mitochondria, which has a function in apoptosis [49-51].

Somayajulu et al. demonstrated that increased insertion of PLP into the mitochondria affects oxidative phosphorylation and leads to mitochondrial dysfunction [39]. By demonstrating the overexpression of PLP1 in COS-7 cells and in PIp 1 transgenic mice, the authors showed that PLP1 translocates to mitochondria by specific cysteine motifs in the N-terminus. However, they did not explore the role of ER in mitochondrial dysfunction. As the ER is a major organelle for protein translation and processing, our study provides novel insight into the association of the ER and mitochondria through MAM function. In Fig. 5, we summarize the pathways in both healthy and PLP1 duplication fibroblasts: overexpression of PLP1 mainly colocalizes with the ER, leading to abnormal ER structures; next, MAM dysfunction may trigger mitochondrial $\mathrm{Ca}^{2+}$ overloading and membrane permeabilization, ultimately leading to mitochondrial dysfunction.

The novel pathway was derived by comparing control and patient fibroblasts, and the differences between the patients' cells were not statistically significant, while the patients were representative of the two PMD subtypes. For the mitochondrial respiration function analysis, an abnormal ER and mitochondria morphology or mitochondrial length, there were no significant differences among the three patients. Fibroblasts of Pt2, who was diagnosed with the connatal type (the most severe type on clinical examination), had the lowest level of mitochondrial maximum respiration (Fig. 1d), the shortest mitochondrial length (Fig. 4c), and the highest ER sheet proportion in the periphery among the three patients' cells (Fig. 2c). Although there were no significant differences among these three patients (Fig. 1d, 2c, 2d, 3b, 4c), these trends may be indicative of mitochondrial dysfunction and PMD clinical severity. The goal of this paper was to investigate the ER-mitochondrion interface in healthy and PLP1duplicated fibroblasts; the connection between the risk of mitochondrial dysfunction and clinical severity will need to be explored in future studies with larger sample sizes.

As a major limitation, our control samples were from a 27-year-old healthy adult, which were not age- and number-matched to our experimental samples of the three children PMD patients. The primary concern was that sampling fibroblasts was an invasive procedure. While the sampling procedure was approved by the upper limb, possibly leading to bleeding 
and scar formation. While a study demonstrated that mitotic activity of fibroblasts was independent of the subject age [52] by measuring ratio of in vivo proportion of progenitor fibroblasts (MF) and functional postmitotic fibrocytes (PMF), the age-related cell stress can affect the ER and mitochondrial morphology, further altering the MAMs function. Consequently, age-related cell stress can affect the distributions of mitochondrial morphology (Fig. 4a). Therefore, for future work, biomarkers that measure the growth, differentiation, and apoptosis of fibroblasts such as the $\mathrm{pH} 6$ activity of $\beta$-galactosidase or TGF- $\beta 1$ will be needed [53]. For another, in this study, we demonstrated that in PLP1 overexpressed cells, closer ERmitochondria interfaces led to pathological mitochondrial morphology, as mediated by MAMs' structure change (Fig. 3). To detail the roles of MAM proteins, future work will be needed to study MAMs-related proteins such as FUNDC1 [42]. In addition, we proposed that mitochondrial $\mathrm{Ca}^{2+}$ overloading could underlie mitophage as a result of PLP1 overexpression (Fig. 5). To investigate this pathway, the formation of ER-mitochondria $\mathrm{Ca}^{2+}$ tunnel proteins [22] will need to be examined. An example protein is $\mathrm{IP}_{3}$, which interacts with the voltage-dependent anion-selective channel across the ER and mitochondria.

\section{Conclusion}

In summary, we demonstrated that PLP1 duplication patients' fibroblasts had altered ER morthology and MAMs structure, leading to abnormal mitochondrial morphology and dysfunction. Our study provided novel insights into the interactions between ER and mitochondria and potential pathogenicity of mitochondria dysfunction resulted from PLP1 duplication mutations in PMD Patients. Moreover, the MAM-associated protein has been suggested as a potential therapeutic target for Alzheimer's disease in Hedskog et al. study [51]. Based on these results, we suggest MAMs as a potential drug target for PMD patients of $P L P 1$ duplication mutant.

\section{Declarations}

\section{Compliance With Ethical Standards}

\section{Ethical Approval}

All experimental procedure were approved by the local ethical committees of Peking University First Hospital.

\section{Consent for Publication}

The authors affirm that human research participants provided informed consent for publication of the images in Fig.S1.

\section{Competing Interests}

The authors declare that they have no competing interests. 
Our study was supported National Natural Science Foundation of China (Grant Number 81271257 to J.W.), and Natural Science Foundation of Beijing (Grant Number 7132208 to J.W.). National Key Research and Development Program of China (No. 2016YFC1306201 and No. 2016YFC0901505). The funders had no role in study design, data collection and analysis, decision to publish, or preparation of the manuscript.

\section{Author contributions}

X. Z., J. W. and L. C. conceived and supervised the research. X. Z. and R.D. designed, conducted and analyzed the SD-SIM imaging data. R. D. performed all the other experiments, analyzed the data and prepared the figures. L. L., S. X., M. H., provided with the mitochondria and ER probes. and helped in some experiments. H.Yan., H.J., B.C., K.G. and J.W. helped with patients recruiting and sample taking; they also helped with the patients' clinical and genetic diagnoses; J.W. helped with image reconstruction. R.D., X.Z., J.W.and L.C. participated in the discussion during the development of this paper. R.D., X. Z., J. W. and L.C. organized and wrote the manuscript. They participated in the discussions, data interpretation and presentation.

\section{Acknowledgements}

We thank Prof. Cheng and Dr. Wu for providing the Seahorse XF instrument and their kindly help in the experiment. We also thank Si Jia Li from the University of Washington for discussing and editing the manuscript. We are grateful for the mCherry-Sec61 $\beta$ kindly supplied by Prof. Gia Voeltz from the university of Colorado. We also thank for all the patients and their families for participation in our study.

\section{References}

1. Hobson GM, Garbern JY (2012) Pelizaeus-Merzbacher disease, Pelizaeus-Merzbacher-like disease 1, and related hypomyelinating disorders. Seminars in neurology 32 (1):62-67. doi:10.1055/s-00321306388

2. Ji H, Li D, Wu Y, Zhang Q, Gu Q, Xie H, Ji T, Wang H, Zhao L, Zhao H, Yang Y, Feng H, Xiong H, Ji J, Yang Z, Kou L, Li M, Bao X, Chang X, Zhang Y, Li L, Li H, Niu Z, Wu X, Xiao J, Jiang Y, Wang J (2018) Hypomyelinating disorders in China: The clinical and genetic heterogeneity in 119 patients. PloS one 13 (2):e0188869. doi:10.1371/journal.pone.0188869

3. Cerghet M, Bessert DA, Nave KA, Skoff RP (2001) Differential expression of apoptotic markers in jimpy and in Plp overexpressors: evidence for different apoptotic pathways. Journal of neurocytology 30 (9-10):841-855. doi:10.1023/a:1019697506757

4. Huttemann M, Zhang Z, Mullins C, Bessert D, Lee I, Nave KA, Appikatla S, Skoff RP (2009) Different proteolipid protein mutants exhibit unique metabolic defects. ASN neuro 1 (3). doi:10.1042/an20090028

5. Zheng X, Duan R, Li L, Xing S, Ji H, Yan H, Gao K, Wang J, Wang J, Chen L (2020) Live-cell 
Science Bulletin 65. doi:10.1016/j.scib.2020.08.016

6. Sistermans EA, de Coo RF, De Wijs IJ, Van Oost BA (1998) Duplication of the proteolipid protein gene is the major cause of Pelizaeus-Merzbacher disease. Neurology 50 (6):1749-1754. doi:10.1212/wnl.50.6.1749

7. Inoue K, Osaka H, Imaizumi K, Nezu A, Takanashi J, Arii J, Murayama K, Ono J, Kikawa Y, Mito T, Shaffer LG, Lupski JR (1999) Proteolipid protein gene duplications causing Pelizaeus-Merzbacher disease: molecular mechanism and phenotypic manifestations. Ann Neurol 45 (5):624-632

8. Regis S, Grossi S, Corsolini F, Biancheri R, Filocamo M (2009) PLP1 gene duplication causes overexpression and alteration of the PLP/DM20 splicing balance in fibroblasts from PelizaeusMerzbacher disease patients. Biochimica et biophysica acta 1792 (6):548-554. doi:10.1016/j.bbadis.2009.04.002

9. Ruiz M, Bégou M, Launay N, Ranea-Robles P, Bianchi P, López-Erauskin J, Morató L, Guilera C, Petit B, Vaurs-Barriere C, Guéret-Gonthier C, Bonnet-Dupeyron MN, Fourcade S, Auwerx J, Boespflug-Tanguy O, Pujol A (2018) Oxidative stress and mitochondrial dynamics malfunction are linked in PelizaeusMerzbacher disease. Brain pathology (Zurich, Switzerland) 28 (5):611-630. doi:10.1111/bpa.12571

10. Miyamoto Y, Eguchi T, Kawahara K, Hasegawa N, Nakamura K, Funakoshi-Tago M, Tanoue A, Tamura H, Yamauchi J (2015) Hypomyelinating leukodystrophy-associated missense mutation in HSPD1 blunts mitochondrial dynamics. Biochemical and biophysical research communications 462 (3):275-281. doi:10.1016/j.bbrc.2015.04.132

11. Scorrano $L$ (2009) Opening the doors to cytochrome c: changes in mitochondrial shape and apoptosis. Int J Biochem Cell Biol 41 (10):1875-1883. doi:10.1016/j.biocel.2009.04.016

12. Rizzuto R, Bernardi P, Pozzan T (2000) Mitochondria as all-round players of the calcium game. The Journal of physiology 529 Pt 1:37-47. doi:10.1111/j.1469-7793.2000.00037.x

13. Ernster L, Schatz G (1981) Mitochondria: a historical review. The Journal of cell biology 91 (3 Pt 2):227s-255s. doi:10.1083/jcb.91.3.227s

14. Dhaunchak AS, Nave KA (2007) A common mechanism of PLP/DM20 misfolding causes cysteinemediated endoplasmic reticulum retention in oligodendrocytes and Pelizaeus-Merzbacher disease. Proceedings of the National Academy of Sciences of the United States of America 104 (45):1781317818. doi:10.1073/pnas.0704975104

15. Inoue K (2019) Pelizaeus-Merzbacher Disease: Molecular and Cellular Pathologies and Associated Phenotypes. Adv Exp Med Biol 1190:201-216. doi:10.1007/978-981-32-9636-7_13

16. Area-Gomez E, Del Carmen Lara Castillo M, Tambini MD, Guardia-Laguarta C, de Groof AJ, Madra M, Ikenouchi J, Umeda M, Bird TD, Sturley SL, Schon EA (2012) Upregulated function of mitochondriaassociated ER membranes in Alzheimer disease. The EMBO journal 31 (21):4106-4123. doi:10.1038/emboj.2012.202

17. Lewis SC, Uchiyama LF, Nunnari J (2016) ER-mitochondria contacts couple mtDNA synthesis with mitochondrial division in human cells. Science (New York, NY) 353 (6296):aaf5549. 
18. Scheper GC, van der Klok T, van Andel RJ, van Berkel CG, Sissler M, Smet J, Muravina TI, Serkov SV, Uziel G, Bugiani M, Schiffmann R, Krageloh-Mann I, Smeitink JA, Florentz C, Van Coster R, Pronk JC, van der Knaap MS (2007) Mitochondrial aspartyl-tRNA synthetase deficiency causes leukoencephalopathy with brain stem and spinal cord involvement and lactate elevation. Nature genetics 39 (4):534-539. doi:10.1038/ng2013

19. de Brito OM, Scorrano L (2010) An intimate liaison: spatial organization of the endoplasmic reticulum-mitochondria relationship. The EMBO journal 29 (16):2715-2723.

doi:10.1038/emboj.2010.177

20. Filadi R, Theurey P, Pizzo P (2017) The endoplasmic reticulum-mitochondria coupling in health and disease: Molecules, functions and significance. Cell calcium 62:1-15. doi:10.1016/j.ceca.2017.01.003

21. Friedman JR, Webster BM, Mastronarde DN, Verhey KJ, Voeltz GK (2010) ER sliding dynamics and ER-mitochondrial contacts occur on acetylated microtubules. The Journal of cell biology 190 (3):363375. doi:10.1083/jcb.200911024

22. Csordás G, Renken C, Várnai P, Walter L, Weaver D, Buttle KF, Balla T, Mannella CA, Hajnóczky G (2006) Structural and functional features and significance of the physical linkage between ER and mitochondria. The Journal of cell biology 174 (7):915-921. doi:10.1083/jcb.200604016

23. Schermelleh L, Ferrand A, Huser T, Eggeling C, Sauer M, Biehlmaier O, Drummen GPC (2019) Superresolution microscopy demystified. Nature cell biology 21 (1):72-84. doi:10.1038/s41556-018-0251-8

24. Sigal YM, Zhou R, Zhuang X (2018) Visualizing and discovering cellular structures with superresolution microscopy. Science (New York, NY) 361 (6405):880-887. doi:10.1126/science.aau1044

25. Zhang L, Wang J, Zhang C, Li D, Carvalho CMB, Ji H, Xiao J, Wu Y, Zhou W, Wang H, Jin L, Luo Y, Wu X, Lupski JR, Zhang F, Jiang Y (2017) Efficient CNV breakpoint analysis reveals unexpected structural complexity and correlation of dosage-sensitive genes with clinical severity in genomic disorders. Human molecular genetics 26 (10):1927-1941. doi:10.1093/hmg/ddx102

26. Hurst S, Garbern J, Trepanier A, Gow A (2006) Quantifying the carrier female phenotype in PelizaeusMerzbacher disease. Genetics in medicine : official journal of the American College of Medical Genetics 8 (6):371-378. doi:10.1097/01.gim.0000223551.95862.c3

27. Kang F, Zhou M, Huang X, Fan J, Wei L, Boulanger J, Liu Z, Salamero J, Liu Y, Chen L (2019) E-syt1 Re-arranges STIM1 Clusters to Stabilize Ring-shaped ER-PM Contact Sites and Accelerate $\mathrm{Ca}(2+)$ Store Replenishment. Sci Rep 9 (1):3975. doi:10.1038/s41598-019-40331-0

28. Divakaruni AS, Paradyse A, Ferrick DA, Murphy AN, Jastroch M (2014) Analysis and interpretation of microplate-based oxygen consumption and pH data. Methods in enzymology 547:309-354. doi:10.1016/b978-0-12-801415-8.00016-3

29. Hill BG, Dranka BP, Zou L, Chatham JC, Darley-Usmar VM (2009) Importance of the bioenergetic reserve capacity in response to cardiomyocyte stress induced by 4 -hydroxynonenal. Biochem $\mathrm{J} 424$ (1):99-107. doi:10.1042/bj20090934 
30. Hill BG, Benavides GA, Lancaster JR, Jr., Ballinger S, Dell'Italia L, Jianhua Z, Darley-Usmar VM (2012) Integration of cellular bioenergetics with mitochondrial quality control and autophagy. Biological chemistry 393 (12):1485-1512. doi:10.1515/hsz-2012-0198

31. Müller CB, Enderlein J (2010) Image scanning microscopy. Physical review letters 104 (19):198101. doi:10.1103/PhysRevLett.104.198101

32. York AG, Chandris P, Nogare DD, Head J, Wawrzusin P, Fischer RS, Chitnis A, Shroff H (2013) Instant super-resolution imaging in live cells and embryos via analog image processing. Nature methods 10 (11):1122-1126. doi:10.1038/nmeth.2687

33. Friedman JR, Voeltz GK (2011) The ER in 3D: a multifunctional dynamic membrane network. Trends in cell biology 21 (12):709-717. doi:10.1016/j.tcb.2011.07.004

34. Jian F, Chen D, Chen L, Yan C, Lu B, Zhu Y, Chen S, Shi A, Chan DC, Song Z (2018) Sam50 Regulates PINK1-Parkin-Mediated Mitophagy by Controlling PINK1 Stability and Mitochondrial Morphology. Cell reports 23 (10):2989-3005. doi:10.1016/j.celrep.2018.05.015

35. Brand MD, Nicholls DG (2011) Assessing mitochondrial dysfunction in cells. The Biochemical journal 435 (2):297-312. doi:10.1042/bj20110162

36. Dranka BP, Benavides GA, Diers AR, Giordano S, Zelickson BR, Reily C, Zou L, Chatham JC, Hill BG, Zhang J, Landar A, Darley-Usmar VM (2011) Assessing bioenergetic function in response to oxidative stress by metabolic profiling. Free radical biology \& medicine 51 (9):1621-1635. doi:10.1016/j.freeradbiomed.2011.08.005

37. Fiuza B, Subelzu N, Calcerrada P, Straliotto MR, Piacenza L, Cassina A, Rocha JB, Radi R, de Bem AF, Peluffo G (2015) Impact of SIN-1-derived peroxynitrite flux on endothelial cell redox homeostasis and bioenergetics: protective role of diphenyl diselenide via induction of peroxiredoxins. Free radical research 49 (2):122-132. doi:10.3109/10715762.2014.983096

38. Fern R (2003) Variations in spare electron transport chain capacity: The answer to an old riddle? Journal of neuroscience research 71 (6):759-762. doi:10.1002/jnr.10553

39. Somayajulu M, Bessert DA, Huttemann M, Sohi J, Kamholz J, Skoff RP (2018) Insertion of proteolipid protein into mitochondria but not DM20 regulates metabolism of cells. Neuroscience letters 678:9098. doi:10.1016/j.neulet.2018.05.005

40. Ott C, Ross K, Straub S, Thiede B, Gotz M, Goosmann C, Krischke M, Mueller MJ, Krohne G, Rudel T, Kozjak-Pavlovic V (2012) Sam50 functions in mitochondrial intermembrane space bridging and biogenesis of respiratory complexes. Molecular and cellular biology 32 (6):1173-1188. doi:10.1128/mcb.06388-11

41. Tang J, Zhang K, Dong J, Yan C, Hu C, Ji H, Chen L, Chen S, Zhao H, Song Z (2020) Sam50-Mic19Mic60 axis determines mitochondrial cristae architecture by mediating mitochondrial outer and inner membrane contact. Cell death and differentiation 27 (1):146-160. doi:10.1038/s41418-019-0345-2

42. Wu W, Li W, Chen H, Jiang L, Zhu R, Feng D (2016) FUNDC1 is a novel mitochondrial-associatedmembrane (MAM) protein required for hypoxia-induced mitochondrial fission and mitophagy. 
43. Voeltz GK, Rolls MM, Rapoport TA (2002) Structural organization of the endoplasmic reticulum. EMBO reports 3 (10):944-950. doi:10.1093/embo-reports/kvf202

44. Borgese N, Francolini M, Snapp E (2006) Endoplasmic reticulum architecture: structures in flux. Current opinion in cell biology 18 (4):358-364. doi:10.1016/j.ceb.2006.06.008

45. Shibata Y, Voeltz GK, Rapoport TA (2006) Rough sheets and smooth tubules. Cell 126 (3):435-439. doi:10.1016/j.cell.2006.07.019

46. Shibata Y, Shemesh T, Prinz WA, Palazzo AF, Kozlov MM, Rapoport TA (2010) Mechanisms determining the morphology of the peripheral ER. Cell 143 (5):774-788. doi:10.1016/j.cell.2010.11.007

47. West M, Zurek N, Hoenger A, Voeltz GK (2011) A 3D analysis of yeast ER structure reveals how ER domains are organized by membrane curvature. The Journal of cell biology 193 (2):333-346. doi:10.1083/jcb.201011039

48. Apetri AC, Horwich AL (2008) Chaperonin chamber accelerates protein folding through passive action of preventing aggregation. Proceedings of the National Academy of Sciences of the United States of America 105 (45):17351-17355. doi:10.1073/pnas.0809794105

49. Green DR, Kroemer G (2004) The pathophysiology of mitochondrial cell death. Science (New York, NY) 305 (5684):626-629. doi:10.1126/science.1099320

50. Rizzuto R, Pinton P, Carrington W, Fay FS, Fogarty KE, Lifshitz LM, Tuft RA, Pozzan T (1998) Close contacts with the endoplasmic reticulum as determinants of mitochondrial Ca2+ responses. Science (New York, NY) 280 (5370):1763-1766. doi:10.1126/science.280.5370.1763

51. Csordas G, Renken C, Varnai P, Walter L, Weaver D, Buttle KF, Balla T, Mannella CA, Hajnoczky G (2006) Structural and functional features and significance of the physical linkage between ER and mitochondria. The Journal of cell biology 174 (7):915-921. doi:10.1083/jcb.200604016

52. Nolte SV, Xu W, Rennekampff HO, Rodemann HP (2008) Diversity of fibroblasts-a review on implications for skin tissue engineering. Cells, tissues, organs 187 (3):165-176. doi:10.1159/000111805

53. Mansbridge JN, Hanawalt PC (1988) Role of transforming growth factor beta in the maturation of human epidermal keratinocytes. The Journal of investigative dermatology 90 (3):336-341. doi:10.1111/1523-1747.ep12456286

\section{Figures}



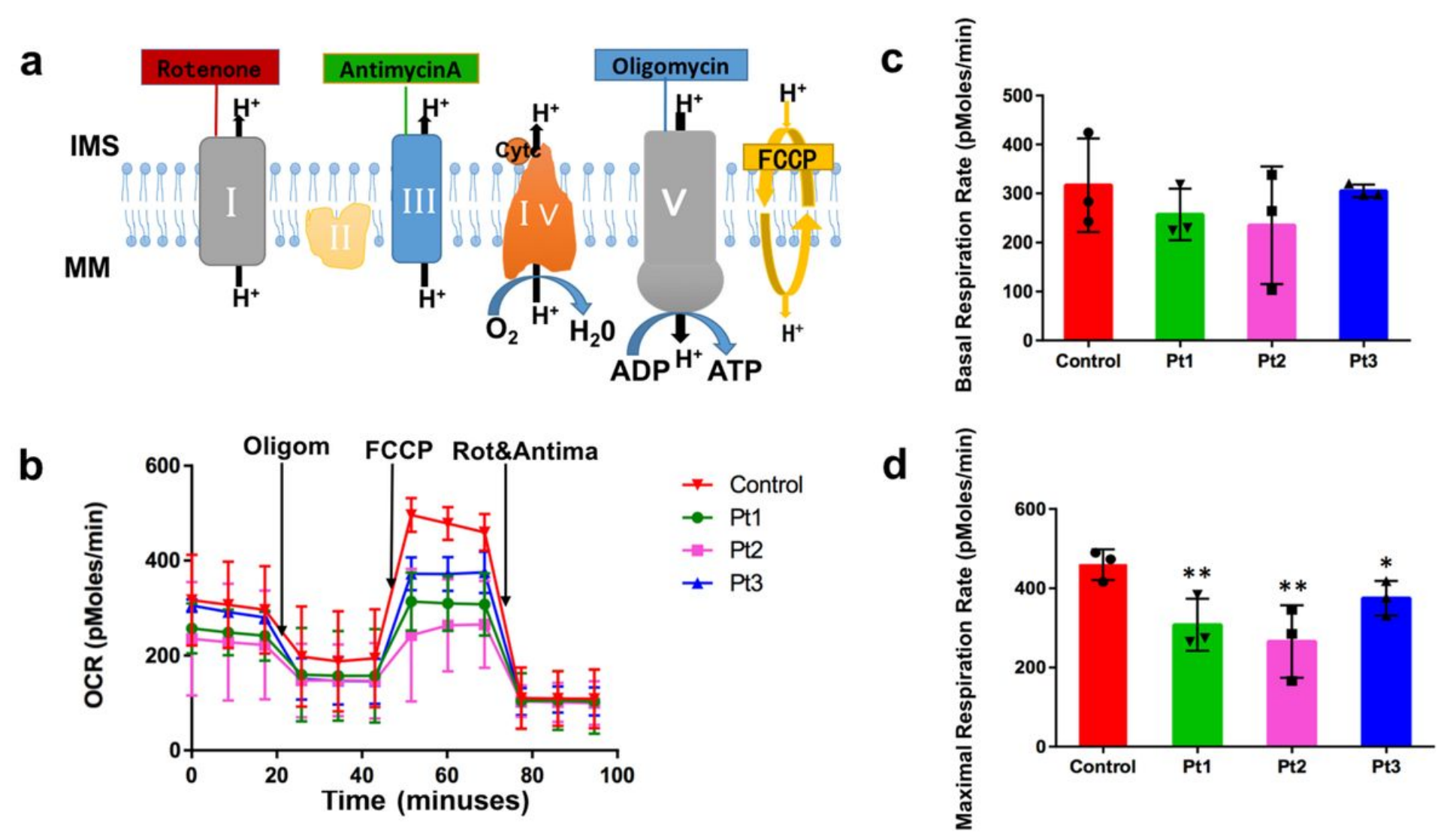

Figure 1

Mitochondrial respiration in the PLP1 duplication and control groups. (a) Mitochondrial respiration initiation drugs along the electron transport chain (ECT), IMS: intermemberane space; MM: mitochondrial matrix. (b) Responses after the addition of ETC modulators at different time points for the PLP1 duplication and control groups. (c) Comparison of the basal respiration rate between the control and patient groups. (d) Comparison of the maximal respiration rate across the control and patient groups. (cd) Error bars represent the mean \pm SD of three independent experiments. Statistical significance was assessed by one-way ANOVA, and significance was set as $<0.05(*),<0.01(* *)$. Oligom: Oligomycin; FCCP: Carbonyl, cyanide-4-(trifluoromethoxy) phenylhydrazone; Rot: Rotenone; AntimA: Antimycin A. 
a

PLP1
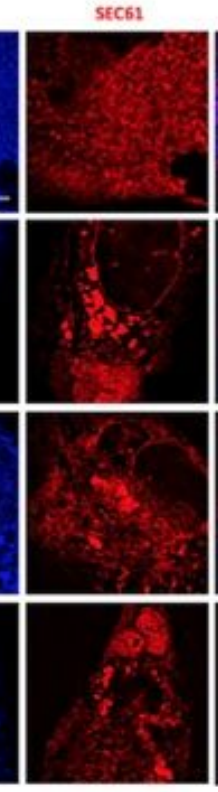

Pt3

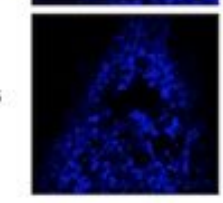

b

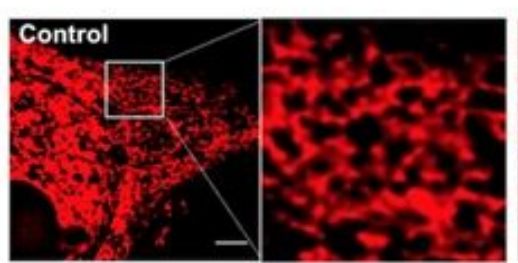

Pt2

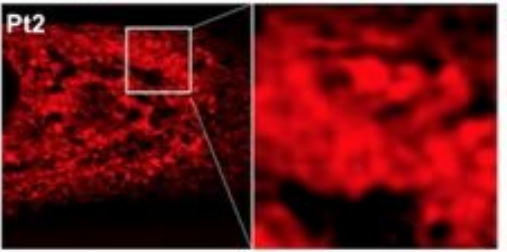

C

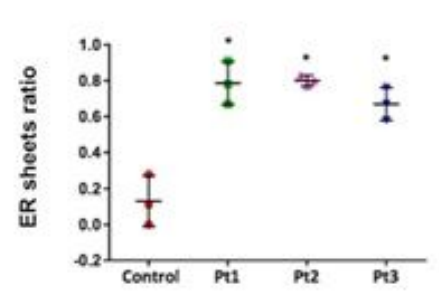

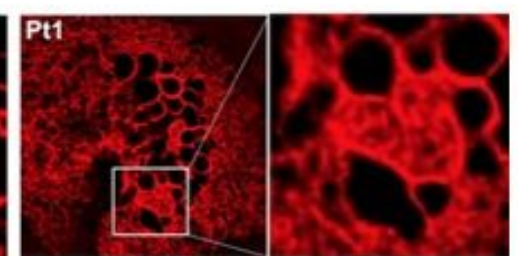

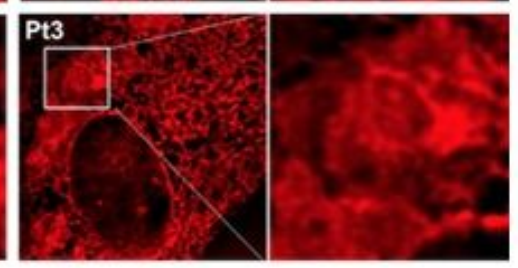

PLP1-5EC61 Merge

Mito-YFP

PLP1-Mito-yFP Merse
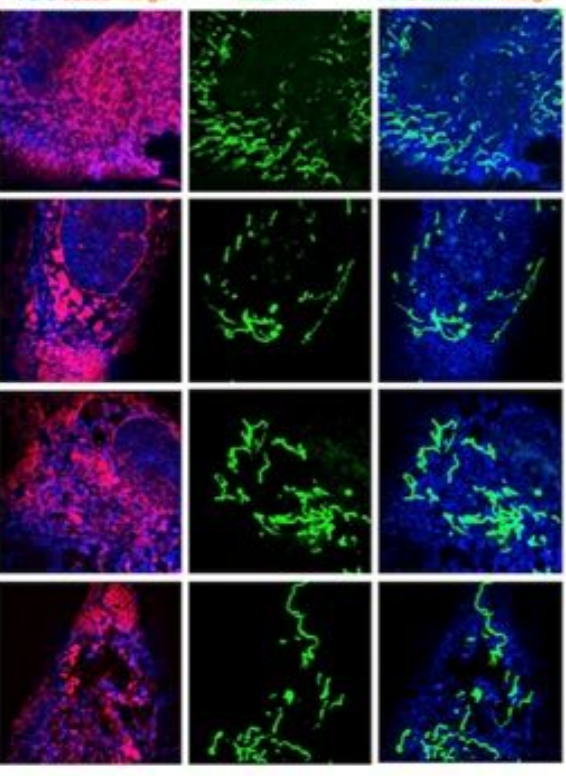

d

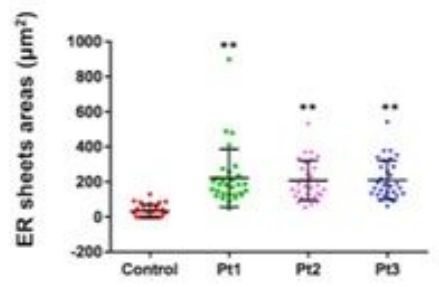

\section{Figure 2}

The subcellular locations of PLP1 in fibroblasts under SD-SIM. (a) The colocalization of PLP1 with the ER and mitochondria. (b) Superresolution live-cell microscopy images of PLP1 duplication patients' ER displayed more "large ER patches"-like sheets in the peripheral section. The "sheet" structure is $5 \mathrm{X}$ enlarged from the white rectangles. (c) The ER sheet ratio was calculated as the proportion of fibroblasts 
independent experiments. (d) The ER sheet area in the control and three patients in the peripheral ER. Error bars represent the means \pm SD of three independent experiments in which 45 fibroblasts were counted. Statistical significance was assessed by the chi-square test (c) and one-way ANOVA (d), and significance was indicated as $<0.05(*),<0.01(* *)$. Scale bars: $5 \mu \mathrm{m}$.

a
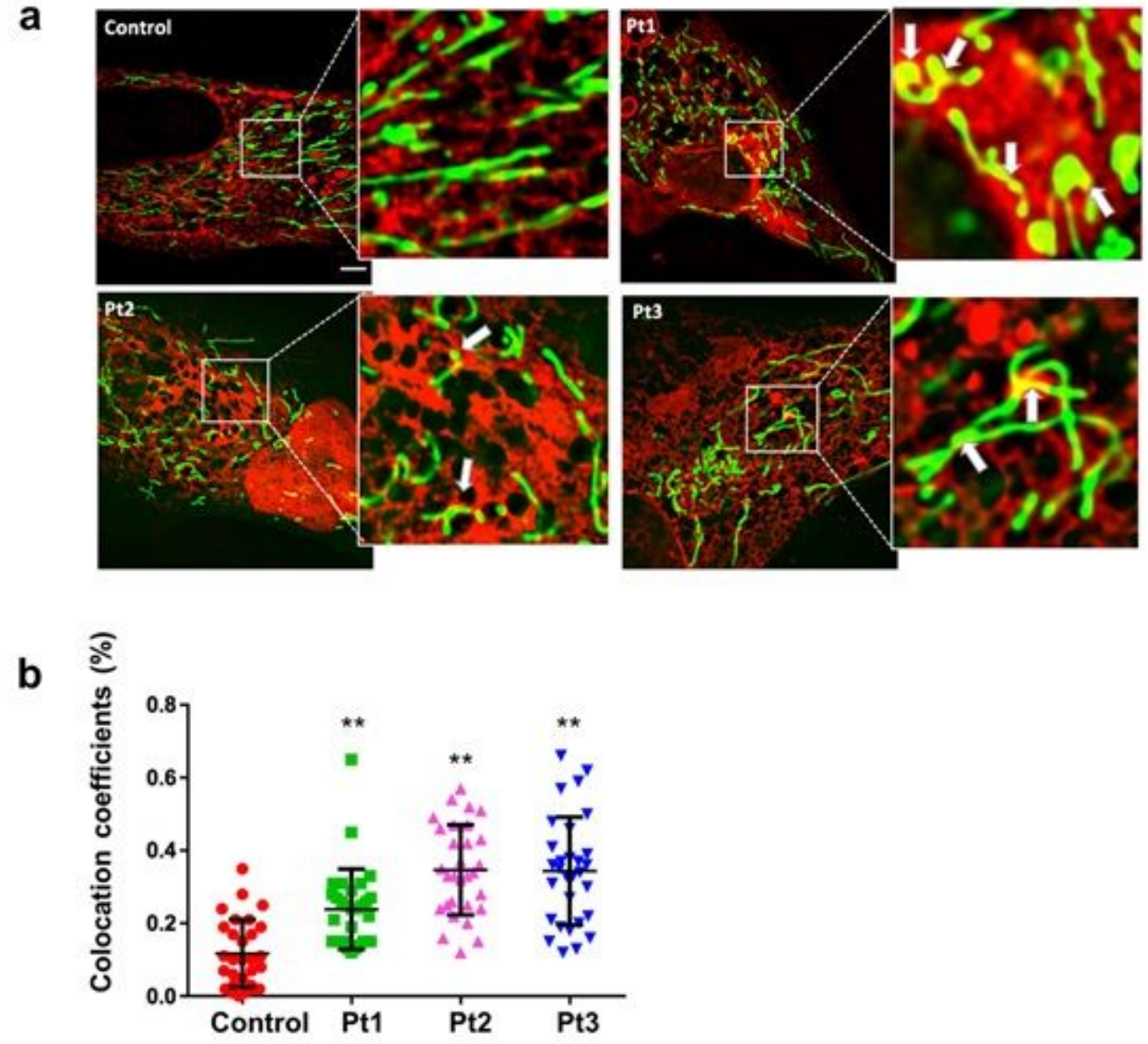

Figure 3

The ER-mitochondria interface in live-cell imaging. (a) White rectangles targeted on the ER sheet peripheral section and $5 \mathrm{X}$ enlarged sections of the white rectangles are shown beside every image. The arrows indicate examples of areas where we measured the colocation coefficients between ER sheets and mitochondria in patient groups. (b) Colocation coefficients showed a significant difference between the PLP1 duplication and control groups (one-way ANOVA, $p<0.01$ ). Thirty areas in each group were counted in three independent experiments. Data represented as the mean $\pm S D$. In the bar charts, significance is indicated as $<0.05\left({ }^{*}\right),<0.01\left({ }^{*}\right)$. Scale bar: $5 \mu \mathrm{m}$. 

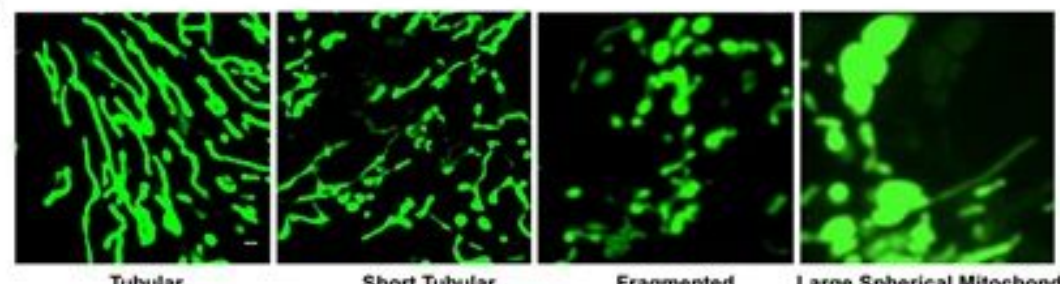

Fragmented

Large Spherical Mitochondria

b

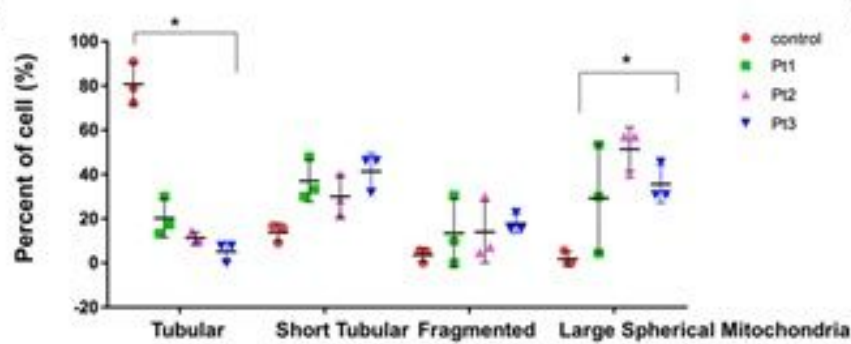

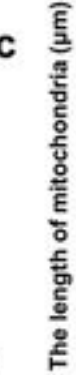

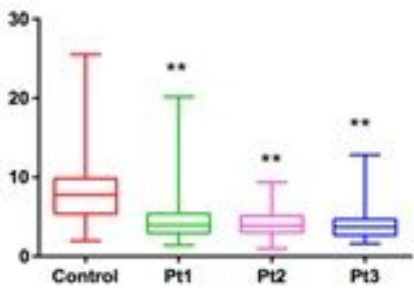

\section{Figure 4}

Mitochondria labeled by Mito-YFP revealed by SD-SIM show a PLP1 duplication leading to abnormal mitochondrial morphology. (a) Four kinds of mitochondrial morphology. (b) Comparison of the distributions of mitochondrial morphology among the three patients and the control fibroblasts. All data represent the mean $\pm S D$ of three independent experiments (20 cells per independent experiment). Statistical significance was assessed by the chi-square test, and significance was set as $<0.05(*),<0.01$ $(\star \star)$. (c) Comparison of mitochondrial length between the patients and controls. We measured 10-15 mitochondrial lengths with a morphology of "Tubular" or "Short Tubular" morphology in each cell, and 4548 cells were counted in 3 independent experiments. For the bar charts, data were analyzed using oneway ANOVA, and significance was set as $<0.05\left(^{(}\right),<0.01{ }^{(*)}$. Scale bar: $1 \mu \mathrm{m}$. 


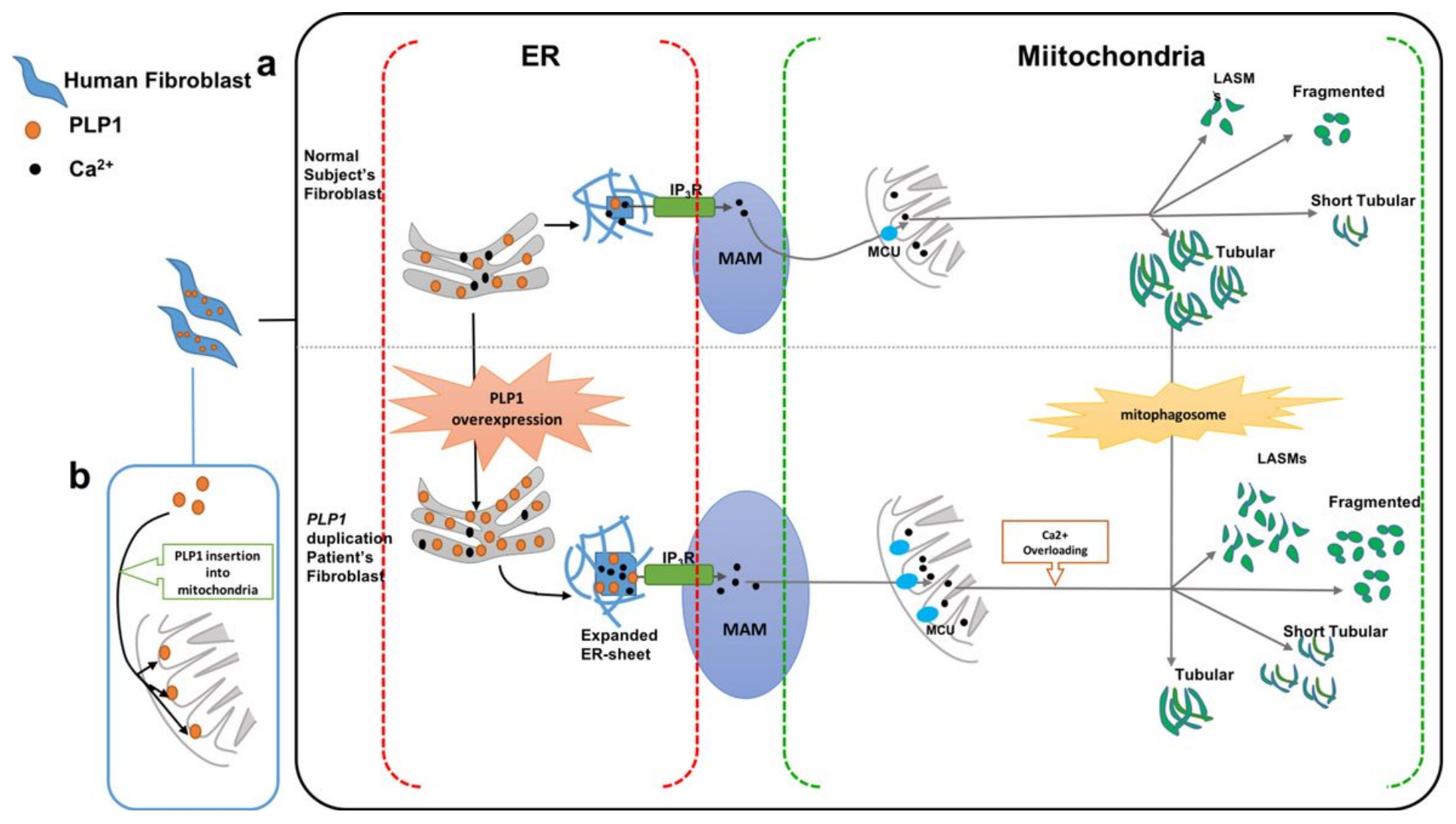

Figure 5

Comparison of the PLP1 cellular pathways in healthy (a, top row) and PLP1-duplicated fibroblasts (b, bottom row). Compared with healthy fibroblasts, PLP1-duplicated cells had larger ER-mitochondrion interfaces near the expanded ER sheets, as evidenced by larger MAMs (blue ovals in the top and bottom rows) and fewer tubular mitochondria but more fragmented, short tubular, and LASMs (relative sizes of these mitochondrial morphologies). Functionally, the greater interfaces in patient fibroblasts might trigger Ca2+ overloading and mitophagosomes, resulting in mitochondrial dysfunction. IP3R: IP3 receptor.

\section{Supplementary Files}

This is a list of supplementary files associated with this preprint. Click to download.

- Supplementarymaterialsrevision.docx 